\title{
Sistemática proposta para seleção de fornecedores em gestão de projetos
}

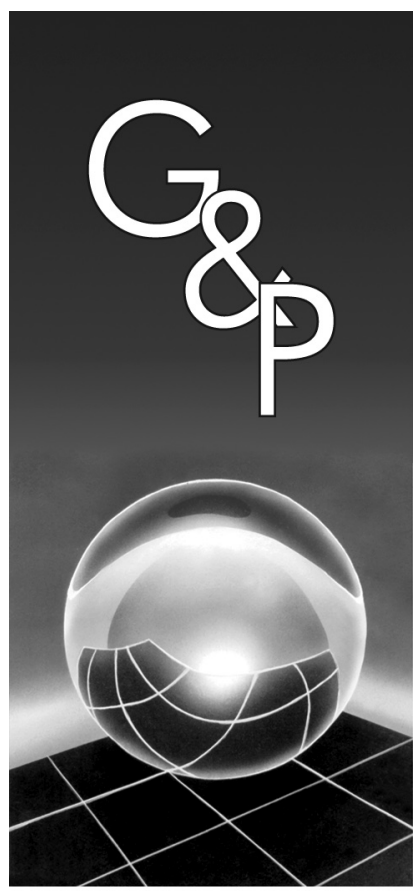

\author{
Luciana Hazin Alencar \\ Adiel Teixeira de Almeida \\ Caroline Maria de Miranda Mota
}

Resumo

A gestão de projetos vem sendo alvo de inúmeros estudos nos últimos anos, uma vez que os projetos estão se tornando cada vez mais complexos. Os fornecedores exercem papéis cruciais na gestão de projetos, estando envolvidos em uma rede de atividades conectadas que, se não desenvolvidas de forma adequada, podem comprometer o sucesso do projeto. Dessa forma, aumenta-se a necessidade de fornecedores qualificados, comprometidos com os objetivos do cliente e capazes de empreender os projetos solicitados. A questão abordada é a seleção de fornecedores para um empreendimento que atenda aos múltiplos objetivos dos decisores (representantes do cliente), de modo que desenvolvam um trabalho conjunto, reduzindo custos e promovendo melhorias nos processos construtivos, assegurando, a qualidade do produto final. Diante da complexidade do problema, propõe-se uma sistemática para seleção de fornecedores, que considera o processo de seleção integrada da equipe do projeto, mediante a revisão bibliográfica realizada.

Palavras-chave: Gestão de projetos. Seleção de fornecedores. Decisão multicritério.

\section{Introdução}

A gestão de projetos vem sendo alvo de inúmeros estudos nos últimos anos, uma vez que os projetos estão se tornando cada vez mais complexos. Dessa forma, aumenta-se a necessidade de fornecedores qualificados, comprometidos com os objetivos do cliente e que sejam capazes de empreendê-los. Tais fornecedores, sejam eles contratantes, subcontratados ou projetistas, exercem papéis cruciais na gestão de projetos, estando envolvidos em uma rede de atividades conectadas que, se não forem desenvolvidas de forma adequada, podem comprometer o sucesso do projeto. Esses fornecedores estão presentes em todo o ciclo de vida do projeto, não necessariamente de forma simultânea em todas as suas etapas, e são responsáveis pelo seu planejamento, execução e acompanhamento.

Para fim de contextualização do problema de seleção de fornecedores na gestão de projetos, escolheu-se o ambiente da construção civil.

A seleção de fornecedores em um projeto é um evento decisivo para o seu sucesso. Várias metodologias relacionadas à seleção de contratantes, projetistas e subcontratadas têm sido desenvolvidas nos últimos anos, o que mostra a importância do tema.
No setor privado, o processo de seleção de fornecedores para clientes privados ocorre de forma desestruturada, além de ser, em geral, baseado na proposta de menor preço, ocasionando sérios problemas de diversas naturezas no decorrer do projeto. Daí a importância do enfoque deste estudo ser direcionado para clientes privados.

É importante esclarecer que neste trabalho são utilizados os termos gestão de projetos e gestão de empreendimentos. A gestão de projeto é abordada na literatura como sistema de produção do tipo grandes projetos (GAITHER; FRAZIER, 2001; SLACK et al., 1995), além de ser um termo mundialmente empregado (project management) e bastante disseminado por diversas instituições. No entanto, no contexto da construção civil, o termo gestão de empreendimentos é bastante utilizado, sendo, portanto, empregado neste trabalho principalmente quando é necessário estabelecer a distinção entre empreendimentos e projetos de engenharia.

Dessa forma, este trabalho apresenta uma sistemática proposta para a seleção de fornecedores para projetos do setor privado, utilizando a modelagem de apoio multicritério à decisão.

Este trabalho está estruturado em cinco seções: a seção 1 contextualiza a gestão de projetos com ênfase na 
seleção de fornecedores, também definindo o objetivo do trabalho; a seção 2 apresenta a metodologia empregada; nas seções 3 e 4, as questões gerais inerentes ao gerenciamento de projeto e ao apoio multicritério à decisão são desenvolvidas, respectivamente; a seção 5 apresenta uma sistemática proposta para a seleção de fornecedores de projetos do setor privado; finalmente, na seção 6 , são apresentadas as conclusões do estudo.

\section{Metodologia}

Este trabalho utilizou a metodologia para a modelagem de um problema no âmbito da Pesquisa Operacional apresentada por Ackoff e Sasieni (1975) e destaca o uso de uma das cinco fases que compõem esta metodologia: a estruturação do problema.

A estruturação ou definição do problema, de acordo com Bidigoli (1989), é uma das etapas mais importantes. Nela, os objetivos, os cursos alternativos de ação e as restrições do problema em questão devem ser bem estabelecidos. Vários métodos de estruturação de problemas têm sido desenvolvidos com o objetivo de apoiar grupos a entrarem em acordo sobre o foco de um problema (ROSENHEAD; MINGERS, 2004; ROSENHEAD, 2006).

No que diz respeito à validação, ela pode ser objetiva ou subjetiva. Uma das formas da validação subjetiva, a utilizada neste trabalho, é a realizada por análise de especialistas, que fazem o julgamento do modelo de acordo com a coerência associada ao sistema em estudo (BANKS, 1998). Dessa forma, utilizou-se uma amostra por conveniência composta por cinco especialistas, que, com base em suas experiências, julgaram de forma positiva a coerência da proposta conceitual realizada neste trabalho. Este procedimento é sugerido por Law e Kelton (1991).

\section{Gestão de Projetos}

O gerenciamento de projetos tem sido estudado por duas associações bem conhecidas por profissionais que atuam nessa área, o PMI (Project Management Institute) e o IPMA (International Project Management Association), além de outras associações, em geral relacionadas à Engenharia de Produção, Pesquisa Operacional e Management Science, tais como: INFORMS, Decision Science Institute e IFORS. Observa-se que as duas primeiras associações desenvolveram documentações que servem de suporte para os gerentes de projetos.

De acordo com o BSI (2000), a gestão de projeto pode ser definida como "o planejamento, monitoramento e controle de todos os aspectos de um projeto e a motivação de todos aqueles envolvidos em alcançar os objetivos do projeto no tempo, custo, qualidade e performance desejada".
É importante esclarecer que o gerenciamento de projetos é executado em um ambiente mais amplo que o do projeto propriamente dito. É necessário que a equipe de gerenciamento entenda esse contexto de maneira que possa definir as fases do ciclo de vida, assim como os processos, as ferramentas e técnicas adequadas ao projeto (PMI, 2004).

Do ponto de vista do cliente, o ciclo de vida de um projeto se inicia quando há um reconhecimento formal dos objetivos do projeto; é por meio da consecução desses objetivos, que se dá sua finalização.

Em geral, o número de fases que compõem o ciclo de vida varia de acordo com a natureza do projeto. De uma forma global, o ciclo de vida inclui fases distintas. A de viabilidade (fase inicial) implica a identificação da necessidade do projeto. É também o momento em que as estratégias de condução são identificadas e selecionadas, e o seu escopo definido. A de planejamento, que ocorre antes de se iniciar o desenvolvimento do projeto. As de execução e controle, praticamente simultâneas, que englobam a materialização do planejamento e o seu acompanhamento; a de finalização, que avalia o resultado do projeto junto ao cliente.

É válido ressaltar que cada etapa do ciclo de vida de um projeto pode ser gerenciada por pessoas diferentes e não necessariamente toda a equipe do projeto estará envolvida desde a etapa inicial até a final.

De acordo com Fewings (2005), o ciclo de vida da construção civil apresenta as seguintes fases: estudo prévio, estratégia, pré-construção, construção, regulamentação e finalização. As duas primeiras fases seriam equivalentes à de viabilidade do projeto; a fase seguinte seria equivalente à de planejamento; a de construção e regulamentação, às fases de execução e controle; sendo a última, à de finalização.

Este trabalho enfoca a fase de pré-construção, que é tratada sob a ótica daqueles projetos que necessitam realizar licitação, seja pública ou privada, para compor a equipe e, conseqüentemente, atingirem o seu objetivo final. Os principais conceitos e trabalhos desenvolvidos relacionados à seleção de fornecedores, à parceria e à engenharia simultânea são abordados a seguir.

\subsection{Seleção de fornecedores}

Smith (1991) afirma existirem dois métodos para seleção de fornecedores: a licitação competitiva e a negociação. Em ambos os casos, o critério geralmente utilizado para seleção é o preço.

"A licitação destina-se a garantir a observância do princípio constitucional da isonomia e a selecionar a proposta mais vantajosa para a administração, sendo processada e julgada em estrita conformidade com os princípios básicos da legalidade, da impessoalidade, da moralidade, 
da igualdade, da publicidade, da integridade administrativa, da vinculação ao instrumento convocatório, do julgamento objetivo e dos que lhes são correlatos" (BRASIL, 1993).

No Brasil, a licitação pública é regida pela Lei 8.666/93 (alterada pelas Leis 8.883/94 e 9.648/98). As empresas privadas, por sua vez, realizam a licitação privada, escolhendo o procedimento mais conveniente à sua realidade, variando de empresa para empresa e do porte e especificidade do projeto a ser empreendido.

A qualificação pode ser dada antes da avaliação (pré-qualificação) ou após a seleção (pós-qualificação). Muitas vezes, um sistema de pós-qualificação é preferível por demandar menos esforço da equipe de avaliação do processo seletivo, uma vez que eles só irão qualificar $\mathrm{a}(\mathrm{s})$ menor(es) proposta(s). Um aspecto negativo desse sistema está relacionado ao tempo e esforço despendido no processo licitatório.

Em relação à pré-qualificação, a desvantagem está associada ao tempo despendido no processo de avaliação. No entanto, as suas vantagens compensam esse aspecto. São elas (JAAFARI; SCHUB, 1990):

a) os contratantes desqualificados não têm o custo associado com a preparação do processo de seleção;

b) os contratantes qualificados são encorajados a participar do processo, uma vez que estarão competindo com poucas empresas, de padrão similar;

c) todos os contratantes que participam do processo já estão qualificados; assim, o cliente pode se concentrar mais na avaliação das propostas do que na avaliação dos contratantes; e

d) conflito em relação ao sistema de pós-qualificação é minimizado, uma vez que neste último são considerados elementos subjetivos, havendo a possibilidade de conflito no caso de uma proposta de menor preço não ser a aceita.

Brown (2003) lista três fatores associados com o procedimento de seleção baseado no menor preço:

a) não há relacionamento estabelecido entre uma proposta de menor preço e um baixo custo final do projeto;

b) a proposta com preço mais baixo pode ser baseada em uma falta de entendimento do projeto; $\mathrm{e}$

c) a menor proposta pode não ser a mais real.

No que diz respeito à seleção de construtoras, várias e significantes pesquisas têm sido desenvolvidas. A maior parte da literatura existente neste assunto diz respeito ao estágio de pré-qualificação, analisando-se, em geral, os critérios para esse processo e a atribuição de pesos, finalizando com casos de aplicação (LO et al., 1998; PONGPENG; LISTON, 2003; HATUSH; SKITMORE 1998; NG; SKITMORE, 1999).

Singh e Tiong (2005) expõem que, na prática, a seleção de construtoras é um complexo problema multicritério de tomada de decisão, em que múltiplos decisores avaliam o desempenho das construtoras em relação a um amplo número de critérios de decisão.

Vários trabalhos foram desenvolvidos no sentido de identificar os critérios de avaliação para o processo de pré-qualificação e seleção de construtoras (PALANEESWARAN; KUMARASWAMY, 2005; PONGPENG; LISTON, 2003; NG; SKITMORE, 1999; TOPCU, 2004).

Palaneeswaran e Kumaraswamy vêm estudando esse processo e evoluindo ao longo dos anos. Em 2001, eles propuseram um modelo universal para pré-qualificação de contratante. O modelo foi desenvolvido com base nas práticas da seleção de contratante de vários projetos públicos desenvolvidos em diferentes países como Canadá, Singapura e Sri Lanka. Em 2003, esses autores propuseram uma estrutura com quatro estágios para obter o "melhor valor" na seleção da construtora. Já em 2005, eles apresentaram um Sistema de Apoio a Decisão (SAD) para pré-qualificação das construtoras.

Shen et al., (2006) desenvolveram um método para avaliar a competitividade das empresas construtoras, identificando os indicadores-chave de competitividade (KCIs) para avaliação dessas empresas no mercado de construção da China. Wong (2004) apresentou um estudo sobre a utilização das preferências do cliente nas avaliações das propostas para prever o desempenho do contratante por meio da abordagem de regressão logística. Zavadskas e Vilutiene (2006) desenvolveram um modelo para seleção de empresas para manutenção de edifícios. Alarcón e Mourgues (2002), por sua vez, propõem um sistema de seleção de construtoras que incorpora a previsão do seu desempenho como um dos critérios para seleção, mediante a análise do seu potencial para alcançar as exigências do cliente.

Vários modelos para seleção de construtoras foram desenvolvidos considerando a abordagem multicritério (HOLT et al., 1993; HOLT, 1994a; 1994b; HATUSH; SKITMORE, 1998; MAHDI et al., 2002; MUSTAFA; RYAN, 1990; ASSAF; JANNADI, 1994; KONG; CHEUNG, 1996; FONG; CHOI, 2000; AL-HARBI, 2001).

Alguns modelos também foram desenvolvidos em relação à seleção de projetistas (HSIEH et al., 2004; NG; CHOW, 2004; CHEUNG et al., 2002) e em relação à seleção de subcontratados (ELAZOUNI; METWALLY, 2000; PING TSERN; LIN, 2002).

No que diz respeito ao processo de seleção integrada, poucos estudos foram desenvolvidos. Ho (2000), em seu estudo, apresentou economias de custo de 11 a $38 \%$ em empresas de construção de Hong Kong, quando o trabalho foi realizado de forma integrada com a equipe do projeto desde o seu início. Rahman e Kumaraswamy (2005) examinaram um conjunto de fatores para seleção 
de projetistas, contratantes, clientes, subcontratados e fornecedores, mediante uma pesquisa realizada com $67 \%$ dos respondentes pertencentes a Hong Kong e os demais pertencentes a diferentes países.

Só um modelo com processo de seleção integrada foi encontrado na literatura. Brown (2003) desenvolveu o project delivery process (PDP), como um meio de promover melhoria contínua e inovação dentro da indústria da construção, mediante a seleção dos fornecedores para um contrato que possuísse a cultura de trabalho cooperativo.

\subsection{Parceria}

A parceria já vem sendo utilizada na indústria de manufatura há algum tempo, com significantes benefícios (BROWN, 2003). A necessidade de uma mudança cultural na indústria da construção civil foi identificada por Latham (1994). Egan (1998) e Byatt (2001), por sua vez, deram prosseguimento ao desenvolvimento de trabalhos nessa linha. Egan (2002) afirma que:

“[...] equipe de trabalho integrada é chave. Times integrados possuem processos mais eficientes e por trabalharem juntos ao longo do tempo podem ajudar a eliminar o antigo estilo da cultura adversária, e começar a gerar projetos mais seguros, com força de trabalho qualificada e treinada".

O aumento da complexidade nas construções e no relacionamento organizacional, a grande oferta de empresas especializadas e a declinante produção da construção têm cultivado uma atmosfera adversa (KUMARASWAMY; MATTHEWS, 2000).

Larson (1995) define parceria como:

"[...] a criação de um relacionamento especial entre partes contratantes. Esse relacionamento encoraja as partes a mudar os seus relacionamentos tradicionais adversários para uma abordagem mais cooperativa, que promova resultados de benefícios mútuos, incluindo a prevenção de maiores disputas".

De acordo com Thomas e Thomas (2005), parceria e equipe integrada são os meios necessários para alcançar o melhor valor em qualquer diretriz que seja definida pelo cliente.

Pesquisas do Instituto da Indústria da Construção dos EUA mostram que a parceria e as alianças estratégicas contribuíram diretamente para o sucesso dos projetos em $80 \%$ dos casos. Em média, empreendimentos que a adotaram obtiveram reduções nas programações na ordem de $15 \%$; reduções nos custos em $12 \%$; e o controle de mudança, segurança e qualidade foram intensificados (CIC, 2005).
É importante que as parcerias promovam a criação de um ambiente de engenharia simultânea, voltado ao aprimoramento dos projetos em todos os seus aspectos.

\subsection{Engenharia simultânea}

Em meio à competitividade no setor produtivo, a engenharia simultânea (concurrent engineering) surgiu como uma alternativa para melhoria dos processos produtivos e de desenvolvimento de produtos (HANNEGHAN et al., 2000). Devido ao sucesso de sua aplicação na manufatura, ela tem sido estendida e aplicada a vários outros setores, principalmente para organizações que trabalham com projetos.

Várias são as definições existentes para a Engenharia Simultânea (ES). No entanto, segundo Rostaldas (1995), todas têm em comum a idéia de que a ES é a execução paralela de diferentes tarefas de desenvolvimento, com equipes multidisciplinares, no intuito de atingir, ao tempo e custo mínimos, um produto ótimo em relação à funcionalidade, qualidade e produtividade.

No contexto da construção civil, pode ser definida como:

“[...] uma tentativa de aprimorar os projetos de engenharia e o processo de construção do empreendimento, para atingir tempos de execução menores e melhoria em custos e qualidade através da integração de projeto, fabricação, construção e outras atividades, maximizando a colaboração e a simultaneidade nas práticas de trabalho" (EVBUOMWAN; ANUMBA, 1998).

Anumba et al., (2002) enfatizam alguns dos benefícios resultantes desse ambiente integrado, tais como: a obtenção do trabalho certo da primeira vez em que é realizado; a satisfação do cliente; a redução do tempo e do custo do desenvolvimento do produto sem o comprometimento da qualidade; a eliminação do desperdício e das mudanças consumidoras de tempo e custo.

Kamara et al., (2001) apontam que as razões para adoção da engenharia simultânea na construção civil baseiam-se:

a) nos benefícios derivados da sua adoção na manufatura - reduções de mais de $70 \%$ no tempo de desenvolvimento do produto;

b) na relevância direta dos seus objetivos e metas com os desafios que estão normalmente presentes na construção civil; e

c) no fato de algumas práticas em construção terem similaridades com a engenharia simultânea.

Assim é possível verificar que a engenharia simultânea tem se desenvolvido ao longo dos anos na construção civil, apresentando efeitos bastante satisfatórios em relação às melhorias que podem ser obtidas em um dado projeto. 


\section{Apoio multicritério à decisão}

A maioria dos problemas econômicos, industriais, financeiros ou políticos são problemas de decisão multicritério. Segundo Roy (1996), decisões são tomadas quando escolhemos fazer ou não fazer algo. Essas decisões relatam os objetivos das organizações, sejam planos de crescimento, implementação de estratégias, desenvolvimento político, dentre outros fatores que podem ser considerados.

$\mathrm{O}$ apoio multicritério à decisão oferece ao decisor algumas ferramentas capazes de torná-lo apto a resolver problemas levando em consideração os mais diversos pontos de vista (VINCKE, 1992).

Os métodos de decisão multicritério não visam encontrar uma solução que seja uma verdade única representada pela ação selecionada, e sim apoiar o processo de decisão. Portanto, tão importante quanto a qualidade da informação disponível é a forma de tratamento analítico aplicada (GOMES, 1998).

Ao modelarmos um problema de decisão multicritério, várias problemáticas podem ser consideradas. Elas podem ser identificadas como de escolha, classificação, ordenação e descrição (ROY, 1996).

Vários métodos multicritério de apoio à decisão são encontrados na literatura. Para Vincke (1992), esse campo de estudo possui duas linhas principais: a Escola Francesa e a Escola Americana.

\section{Sistemática proposta para seleção de fornecedores}

A sistemática proposta para seleção de fornecedores de um projeto é apresentada nesta seção. Baseia-se no modelo desenvolvido por Brown (2003) e adaptado ao contexto do setor privado, agregando também idéias surgidas com base na literatura.

As etapas dessa sistemática podem ser verificadas na Figura 1.

Engloba as etapas de identificação dos objetivos do cliente, seleção de projetista, seleção de construtoras e seleção de subcontratadas. Na primeira etapa, considera-se que o cliente é representado por um grupo de

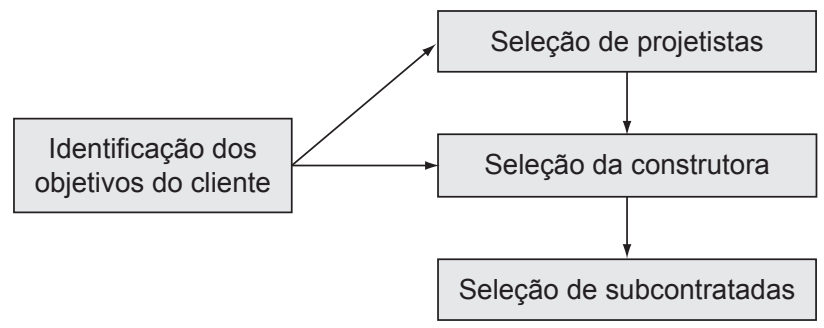

Figura 1. Sistemática proposta para seleção de fornecedores (Fonte: os autores). pessoas. Essas pessoas são os decisores do processo de seleção, sendo também responsáveis pelo acompanhamento do empreendimento até o seu término. Os critérios de decisão são estabelecidos nessa etapa, assim como sua ponderação, considerando as preferências do grupo. É importante a presença do analista, já nessa etapa, desde o início do processo. Na etapa seguinte, ocorre a seleção da empresa de projetistas, considerando as preferências do grupo de decisores. Já na seleção da construtora, as empresas são primeiramente pré-qualificadas, de acordo com as preferências do grupo de decisores. Em um segundo momento, cada uma delas trabalha em conjunto com a empresa de projetistas selecionada e passa por outro estágio de avaliação que permite chegar à seleção final. Já no estágio de seleção de subcontratadas, a empresa construtora selecionada dá início ao processo, listando as atividades que serão subcontratadas. A partir daí, os membros da equipe - representantes do cliente, projetistas e construtor, sugerem as empresas que vão participar do processo de seleção. Este, embora seja estabelecido pelo cliente, leva em consideração as preferências da construtora, uma vez que ela será a responsável pelos subcontratados.

É importante deixar claro que a política de contratação é definida previamente, antes de se dar início ao processo de seleção. Esse processo ocorre na fase de planejamento do ciclo de vida do projeto.

O principal diferencial dessa sistemática em relação aos modelos encontrados na literatura está em considerar a seleção integrada dos fornecedores, ou seja, dos projetistas, do construtor e inclusive dos subcontratados dessa forma, todos são co-responsáveis e co-beneficiados pelos resultados obtidos. Todo o processo de seleção é baseado nos critérios definidos pelos representantes do cliente, tratando suas preferências de forma estruturada. Outro aspecto importante é que os representantes do cliente envolvidos são os que estarão também envolvidos no projeto durante todo o seu ciclo de vida. Não foram encontrados na literatura modelos que apresentassem uma seleção integrada dos três fornecedores.

A seguir, os estágios de identificação dos objetivos do cliente e seleção de construtoras são detalhados.

\subsection{Identificação dos objetivos do cliente}

Nesse estágio, as verdadeiras expectativas e objetivos em relação ao empreendimento são determinados. $\mathrm{O}$ cliente é geralmente representado por um grupo, e não por um só representante, que se constituirá nos decisores do processo de seleção. Todos já estão instruídos com as informações relacionadas ao empreendimento. As etapas desse estágio podem ser verificadas na Figura 2.

Os objetivos definidos nesse estágio são os critérios de decisão, utilizados para a seleção dos projetistas e construtoras. É válido ressaltar que devem ser determinados 
tanto os critérios qualificadores quanto os ganhadores de pedido. O critério qualificador é aquele no qual os objetivos devem estar dentro de um nível determinado, chamado de nível de qualificação, para ser sequer considerado pelo cliente. Abaixo desse nível, a empresa terá prejuízos, pois estará fora de um padrão e não será considerada pelos clientes. $\mathrm{O}$ critério ganhador de pedido é aquele que influencia diretamente no potencial competitivo da empresa. Quando se aumenta o desempenho desses objetivos, aumenta-se significativamente o benefício competitivo. O critério menos importante representa os objetivos que não são significativos para os consumidores e não são identificados nesse trabalho. A diferença entre esses critérios pode ser visualizada na Figura 3.

Os critérios qualificadores são utilizados nos filtros, de forma a eliminar aquelas empresas que não atenderem às exigências mínimas requeridas pelo cliente. Já os ganhadores de pedido são utilizados no estágio de préqualificação e seleção final.

Para identificação dos objetivos, pode-se utilizar o brainstorm, estruturado ou não, para ajudar o grupo a imaginar tantos critérios quanto possível em torno do problema em questão. Entende-se por brainstorm estru-

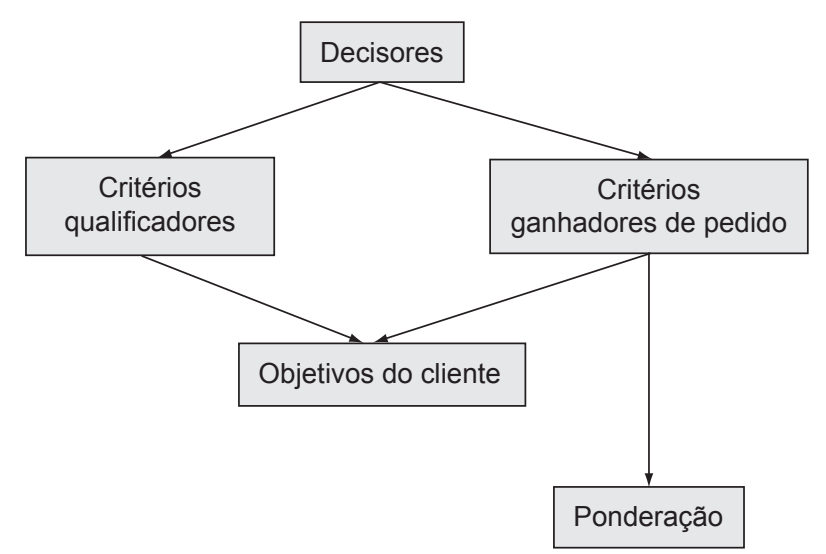

Figura 2. Identificação dos objetivos do cliente (Fonte: os autores). turado quando todas as pessoas do grupo contribuem com sugestões a cada rodada; já, por não estruturado, quando os membros do grupo simplesmente dão idéias conforme elas surgem, criando uma atmosfera mais relaxada (GOMES et al., 2002). É importante a presença de um facilitador ou de uma analista, estimulando sempre os participantes a atuarem no processo. Para auxiliar os decisores, uma lista dos critérios identificados na revisão da literatura lhes é fornecida. Várias rodadas podem ser executadas até que os critérios estejam definidos.

Uma vez definidos os critérios, o próximo estágio é o estabelecimento da importância relativa entre eles. Posteriormente, o edital é elaborado e divulgado, podendo-se dar início ao processo de seleção.

\subsection{Seleção da construtora}

O procedimento para seleção de construtoras é composto pelas etapas apresentadas na Figura 4. São descritas as etapas de reunião de esclarecimento, filtro, pré-qualificação, submissão de propostas de preço, prazo e qualidade, engenharia simultânea e seleção final.

\subsubsection{Reunião de esclarecimento}

Todas as construtoras interessadas no empreendimento são convidadas a participar da reunião de esclarecimento, já com as informações relativas ao tipo do projeto, localização, prazo estimado e outros dados básicos.

Os interessados devem, já nessa reunião, entregar a documentação da empresa a concorrer nesse processo de seleção. Nesse momento, também são apresentados aos licitantes os objetivos do empreendimento e os projetistas já selecionados, além do processo pelo qual será dada a seleção.

\subsubsection{Filtro}

Esse estágio se inicia com a aplicação de um filtro composto pelas questões mandatórias demandadas pelo cliente. Os níveis mínimos aceitáveis de cada atributo são usados para eliminar alternativas inaceitáveis.
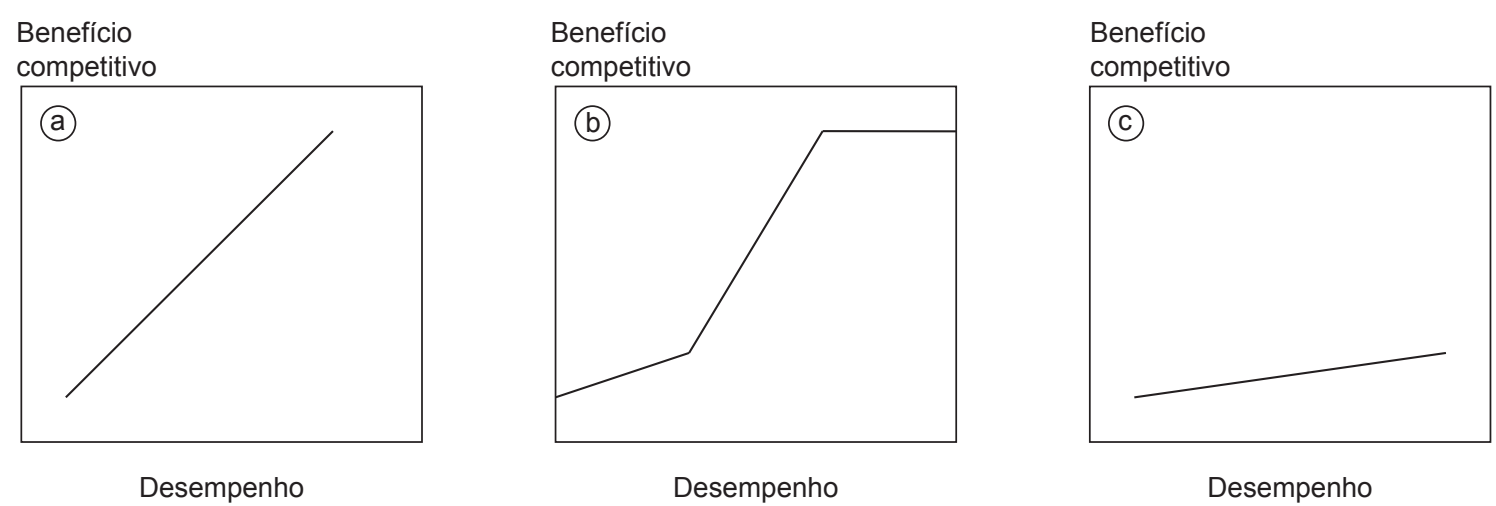

Figura 3. a) Critérios ganhadores de pedido; b) qualificadores; e c) e menos importante (adaptado de SLACK et al., 1995). 


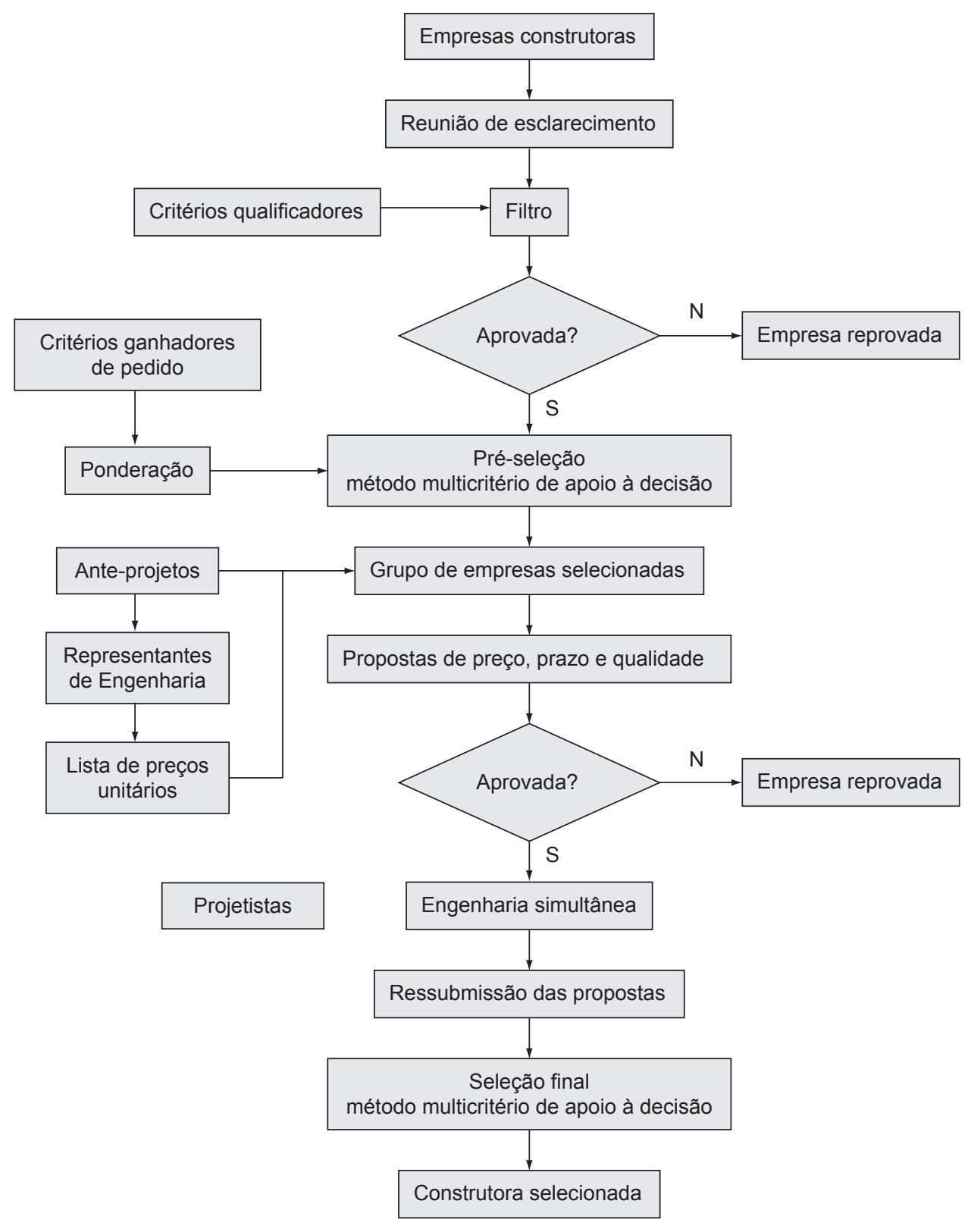

Figura 4. Sistemática para seleção de construtoras (Fonte: os autores).

\subsubsection{Pré-seleção}

Nessa etapa, os decisores, em conjunto com o analista, devem elaborar um questionário que envolva os critérios ganhadores de pedido que não possuam uma mensuração objetiva, definidos em fase anterior. Serão desenvolvidas questões que forneçam informações relevantes para o projeto a respeito da empresa licitante e que contribuam para a sua avaliação aliadas a outro conjunto de informações coletado ao longo do processo.

Dessa forma, cada licitante receberá um questionário, assim como um formulário em que deverá prover informações sobre as pessoas que o responderam . Eles terão um prazo pré-estipulado, em torno de uma semana, para devolver o questionário e o formulário juntos.
Baseado na informação recolhida, as avaliações das empresas licitantes em relação aos critérios definidos são realizadas. Posteriormente aplica-se uma metodologia multicritério de apoio à decisão em grupo. Recomenda-se que 3 (três) construtoras sejam selecionadas e encaminhadas para o próximo estágio, de maneira a não inviabilizar as etapas seguintes.

As empresas pré-selecionadas devem indicar alguns dos seus clientes passados para atuarem como avaliadores. Desses, três são escolhidos e contactados no sentido de esclarecer o processo de seleção em curso e solicitar sua colaboração. Só após sua aceitação será encaminhado um questionário para avaliação das construtoras. 
Muitas vezes, as construtoras, que se submetem ao processo de seleção, já trabalharam com o cliente (seja na empresa local ou na sua filial em outro estado) facilitando a avaliação. Nos casos em que a empresa não tenha ainda trabalhado com esse cliente, o mesmo processo de esclarecimento é realizado, porém com uma ênfase maior na importância de sua participação.

\subsubsection{Submissão de propostas}

Nesse estágio, as construtoras pré-selecionadas participam de uma entrevista, em que devem ser mencionados alguns fatores relevantes para os clientes e alguns aspectos que precisam ser esclarecidos, seja em relação aos questionários ou à avaliação realizada pelos clientes passados. Ainda durante a entrevista, os licitantes são instruídos sobre as demais etapas do processo. Eles recebem os projetos básicos do empreendimento assim como uma planilha de quantidades, que contém a lista dos materiais e atividades a serem realizadas para orçamento.

As propostas são baseadas no custo das quantidades solicitadas, no prazo de conclusão estimado e na qualidade dos materiais e atividades, conforme especificado no documento de licitação entregue. É importante ressaltar que, em geral, o cliente estipula um limite máximo de custo e duração para o empreendimento.

Após o prazo estipulado pelo cliente, as propostas devem ser entregues e avaliadas. As empresas que submeterem as propostas abrangendo todos os itens solicitados são aprovadas nessa fase e passam para a etapa de engenharia simultânea.

\subsubsection{Fase de engenharia simultânea}

Nessa fase, cada uma das construtoras que permaneceram no processo trabalha separadamente com os projetistas. Elas devem subsidiar os projetistas com informações e orientações em relação às soluções cotidianas tratadas nos projetos. O resultado é dado por um projeto básico para cada construtora, adequado ao seu método preferido de trabalho, além do aperfeiçoamento da construtibilidade do empreendimento, melhorando a qualidade e reduzindo os riscos de todas as partes. Os custos de manutenção também devem ser levados em consideração nessa fase.

\subsubsection{Seleção final}

Seguindo o término das tarefas anteriores, solicita-se às construtoras uma revisão nas propostas de custo, prazo e qualidade, baseadas nos projetos básicos desenvolvidos.
A seleção final é feita com base nos critérios de custo, prazo e qualidade. O custo e o prazo são calculados como a redução do orçamento e da duração média do empreendimento, respectivamente, em relação à proposta anterior. Qualidade, por sua vez, é avaliada de acordo com aspectos identificados pelo cliente. As avaliações feitas pelos clientes passados também entram como input nessa avaliação final.

\section{Conclusões}

Haja vista a complexidade do gerenciamento de projetos, que vem aumentando ao longo dos anos, uma ênfase crescente vem sendo dada ao desenvolvimento de novas formas de seleção de fornecedores, no intuito de minimizar conflitos entre os diferentes atores envolvidos, obter melhores relacionamentos entre eles e atender aos objetivos do projeto, geralmente centrados na entrega do produto final ao menor custo possível, com qualidade, respeitando o prazo e o escopo definidos, de forma a obter a satisfação do cliente.

O estudo desenvolvido está relacionado à seleção de fornecedores para um empreendimento do setor privado. Constatou-se, com base na revisão da literatura realizada, que a seleção dos fornecedores é uma etapa decisiva para o sucesso do empreendimento. Dentre os modelos encontrados na literatura, não foram encontrados os que tratassem a seleção com todos os fornecedores integrados, de forma adequada.

A adoção dessa sistemática pode contribuir significativamente para o sucesso do empreendimento. No entanto, é necessário que se disponibilize considerável tempo e custo a serem gastos no processo de seleção, não sendo, portanto, adequado para todos os tipos de projetos. $\mathrm{O}$ modelo é particularmente adequado para empreendimentos grandes e complexos.

É importante ressaltar que embora este estudo esteja contextualizado para a construção civil, a proposta realizada pode ser aplicada a outros ambientes de projetos, realizando-se as devidas adaptações.

Dessa forma, a proposta conceitual desenvolvida possibilita selecionar os fornecedores que apresentam maior compromisso com os objetivos do cliente, incorporando o trabalho integrado como possível veículo redutor de custos e atrasos, além de proporcionar métodos construtivos mais eficientes e conseqüentemente um gerenciamento do empreendimento eficiente e eficaz. 


\title{
Systematic proposal for selecting suppliers in project management
}

\begin{abstract}
The project management has been the target of numerous studies in recent years since projects have become increasingly complex. The suppliers play crucial roles in project management being involved in a network of connected activities, which if not developed appropriatel, can compromise the success of the project. Hence, there has been a growing need for qualified suppliers, who are committed to the client's objectives and are able to undertake the projects requested. The question brought up is the suppliers' selection for a project according to the multiple criteria of the decision makers (clients representation), so they can undertake an integrated work reducing costs and providing improvements in the constructive processes, assuring the quality of the final product. Due to the complexity of the problem, a systematic proposal is put forward for selecting suppliers considering an integrated selection process of the project team based on the literature review carried out.
\end{abstract}

Keywords: Project management. Supplier selection. Multi criteria decision making.

\section{Referências bibliográficas}

ACKOFF, R. L.; SASIENI, M. W. Pesquisa operacional. Tradução José L. Moura; Revisão Antônio de Miranda Netto. Rio de Janeiro: Livros Técnicos e Científicos, 1975.

AL-HARBI, K. M. Application of the AHP in project management. International Journal of Project Management, v. 19, n. 1, p. 19-27, 2001.

ALARCÓN, L. F.; MOURGUES, C. Performance modeling for contractor selection. Journal of management in engineering. v. 18, n. 2, p. 52-60, 2002.

ANUMBA, C. J.; BAUGH, C.; KHALFAN, M. M. Organisational structures to support concurrent engineering in construction, Industrial Management \& Data Systems, v. 102, n. 5, p. 260-70, 2002.

ASSAF, S.; JANNADI, O. M. A multi-criterion decision-making model for contractor prequalification selection. Building research and information. v. 22, n. 6, p. 332-335,1994.

BANKS, J. (ed.) Handbook of simulation - principles, methodology, advances, applications and practice. New York: John Willey \& Sons. 1998.

BIDGOLI, H. Decision support systems - principle and practice. Saint Paul: West Publishing Company. 1989.

BRASIL. Decreto - Lei no 8666 de 21 de junho de 1993 e Leis n $^{\circ} 8883$ de 8 de junho de 1994 e 9648 de 27 de maio de 1998. Ministério da Administração e Reforma do Estado. Brasília, DF. 1998.

BROWN, D. C. A methodology for selecting the construction team. 2003. Tese (Doutorado em Engenharia Civil) Department of Civil and Environmental Engineering. Faculty of Engineering and Applied Science. Southampton, England, 2003.

BSI - BRITISH STANDARD INSTITUTE. Guide to Project Management. BS 6079, 2000. BSI, UK.

BYATT, I. Delivering better services for citizens, 2001, London, UK.

CHEUNG, F.; KUEN, J.; SKITMORE, M.. Multi-criteria evaluation model for the selection of architectural consultants. Construction management and economics. v. 20, n. 7, p. $569-580,2002$.
CIC - Construction Industry Council. Selecting the team. Strategic Forum for Construction, 2005. Annual report. Disponível em: $<\mathrm{http}$ ://www.strategicforum.org.uk>. Acesso em: 11/2005.

EGAN, J. Accelerating change. Rethinking construction. Strategic Forum for Construction, 2002, London.

Rethinking construction. Department of the Environment, Transport and the Regions, 1998, London, UK.

ELAZOUNI, A. M.; METWALLY, F. G. D-SUB: Decision support system for subcontracting construction works. Journal of construction engineering and management. v. 126, n. 3, p. 191-200, 2000.

EVBUOMWAN, N. F. O.; ANUMBA, C. J. An integrated framework for concurrent life-cycle design and construction. Advances in engineering software, v. 29, n. 7-9, p. 587-97, 1998.

FEWINGS, P. Construction Project Management: an integrated approach. New York: Taylor \& Francis, 2005.

FONG, S. W.; CHOI, S. K. Y. Final contractor selection using the analytical hierarchy process. Construction Management and Economics, v. 18, n. 5, p. 547-57, 2000.

GAITHER, N.; FRAZIER, G. Administração de produção e operações. São Paulo: Pioneira, 2001.

GOMES, L. F. A. M. Da informação à tomada de decisão: agregando valor através dos métodos multicritério. Recitec, Recife, v. 2, n. 2, p. 117-139, 1998.

GOMES, L. F. A. M.; GOMES, C. F. S.; ALMEIDA, A. T. de. Tomada de Decisão Gerencial: enfoque multicritério. São Paulo: Atlas, 2002.

HANNEGHAN, M.; MERABTI, M.; COLQUHOUN, G. A viewpoint analysis reference model for Concurrent Engineering. Computers in industry. v. 41, n. 1, p. 35-49, 2000.

HATUSH, Z.; SKITMORE, M. Contractor selection using multicriteria utility theory: an additive model. Building and environment. v. 33, n. 2, p. 105-115, 1998.

HOLT, G. D.; OLOMOLAIYE, P. O.; HARRIS, F. C. A conceptual alternative to current tendering practice. Building research and information. v. 21, n. 3, p. 167-172, 1993.

Evaluating performance potential in the selection of construction contractors. Engineering construction and architectural management. v. 1, n. 1, p. 29-50, 1994a. 
Incorporating project specific criteria and client utility into the evaluation of construction tenderers. Building research and information. v. 22, n. 4, p. 214-21, 1994b.

HO, O. S. Enhancing construction technology through strategic partnering: a contractor's perspective. In: SYMPOSIUM ON QUALITY HOUSING: PARTNERING SYMPOSIUM, 2000, Hong Kong. Anais... Hong Kong. Disponível em: <http://www. info.gov.hk/hd/eng/events/conf00/day1.htm > Data de acesso: 05 fev. 2006.

HSIEH, T. Y.; LU, S. T.; TZENG, G. H. Fuzzy MCDM approach for planning and design tenders selection in public office buildings. International journal of project management. v. 22, n. 7, p. 573-584, 2004.

JAAFARI, A.; SCHUB, A. Surviving failures - lessons from fieldstudy. Journal of construction engineering and management. v. 116, n. 1, p. 68-86, 1990.

KAMARA, J.; ANUMBA, C.; EVBUONWAN, N. Assesing the suitability of current briefing practices in construction within a concurrent engineering framework. International journal of project management. v. 19, n. 6, p. 337-351, 2001.

KONG, W. K.; CHEUNG, S. M. A multi-attribute tender evaluation model. In: CIB W89, Proceedings... Beijing, 21-24 oct. 1996.

KUMARASWAMY, M.; MATHEWS, J. D. Improved subcontractor selection employing partnering principles. Journal of management in engineering, v. 16, n. 3, p. 47-57, 2000.

LARSON, E. Project partnering - results of study of 280 construction projects. Journal of management in engineering, v. 11, n. 2, p. 30-35, 1995.

LATHAM, M. Constructing the team. London, UK: HMSO, 1994.

LAW, A.M.; KELTON, W.D. Simulation model \& analysis. 2. ed. Nova York: McGraw Hill, Inc., 1991.

LO, W.; CHAO, C.; HADAVI, A.; KRIZEK, R. J. Contractor selection process for Taipei mass rapid transit system. Journal of construction and engineering management, v.14, n.3, p.57-65, 1998.

MAHDI, I. M.; RILEY, M. J.; FEREIG, S. M.; ALEX, A. P. A multi-criteria approach to contractor selection, Eng. Constr. Archit. Manage., v. 9, n. 1, p. 29-37, 2002.

MUSTAFA, M. A.; RYAN, T. C. Decision support for bid evaluation. Project management journal. v. 4, n. 8, p. 230-235, 1990.

NG, S. T.; CHOW, L. Framework for evaluating the performance of engineering consultants. Journal of professional issues in engineering education and practice. v. 130, n. 4, p. 280-288, 2004.

NG, S. T.; SKITMORE, R. M. Client and Consultant Perspectives of Prequalification Criteria, Building and Environment, v. 34, n. 5, p. 607-621, sep. 1999.

PALANEESWARAN, E.; KUMARASWAMY, M. Web-Based Advisory Decision Support System for Designer-Builder Prequalification. Journal of Computing in Civil Engineering, v. 19 , n. 1, p. $69-82,2005$.
Recent advances and proposed improvements in contractor prequalification methodologies, Building and Environment, v. 36, n. 1, p. 73-87, 2001.

PING TSERNG, H.; LIN, P. H. An accelerated subcontracting and procuring model for construction projects. Automation in construction. v. 1, n. 11, p. 105-125, 2002.

PMI - Project Management Institute. A guide to the project management body of knowledge - PMBOK GUIDE, 2004. Pennsylvania, EUA.

PONGPENG, J.; LISTON, J. Contractor ability criteria: a view from the Thai construction industry. Construction Management and Economics, v. 21, n. 3, p. 267-282, 2003.

RAHMAN, M. M.; KUMARASWAMY, M.M. Relational selection for collaborative working arrangements. Journal of Construction Engineering and Management, v. 131, n. 10, p. 1087-1098, 2005.

ROSENHEAD, J. Past, present and future of problem structuring methods. Journal of the operational research society. v. 57, n. 7, p. 759-765, 2006.

ROSENHEAD, J.; MINGERS, J. (Eds.). Rational analysis for a problematic world revisited: problem structuring methods for complexity, uncertainty and conflict. 2. ed. New York: Jonh Wiley \& Sons, 2004.

ROSTALDAS, A. Planning and control of concurrent engineering projects. International journal of production economics. v. 38, n. 1, p. 3-13, 1995.

ROY, B. Mulcriteria Methodology for Decision Aiding. Netherlands: Kluwer Academic Plublishers, 1996.

SHEN. L.; LU. W.; YAM. M. Contractor key competitiveness indicators: a china study. Journal of construction engineering and management. v. 132, n. 4, p. 416-424, 2006.

SINGH, D.; TIONG, R. L. A fuzzy decision framework or contractor selection. Journal of Construction Engineering and Management, v. 131, n. 1, p. 62-70, 2005.

SLACK, N. et. al. Administração da produção. São Paulo: Atlas, 1995.

SMITH, R. C. Estimating and tendering for building work. London: Longman scientific and technical, 1991.

THOMAS, G.; THOMAS, M. Construction partnering \& integrated teamworking. Blackwell Publishing: London, 2005.

TOPCU, Y. I. A decision model proposal for construction contractor selection in Turkey. Building and Environment, v. 39, n. 4, p. 469-481, 2004.

VINCKE, P. Multicriteria decision-aid. Bruxelles: Wiley, 1992.

WONG, C. H. Contractor performance prediction model for the United Kingdom construction contractor: study of logistic regression approach. Journal of construction engineering and management. v. 130, n. 5. p. 691-698, 2004.

ZAVADSKAS. E.; VILUTIENE. T. A multiple criteria evaluation of multi-family apartment block's maintenance contractors: I- model for maintenance contractor evaluation and the determination of its selection criteria. Building and environment. v. 41, n. 5, p. 621-632, 2006. 


\section{Sobre os autores}

\section{Luciana Hazin Alencar}

Adiel Teixeira de Almeida

\section{Caroline Maria de Miranda Mota}

Grupo de Pesquisa em Sistemas de Informação e Decisão - GPSID,

Departamento de Engenharia de Produção, Universidade Federal de Pernambuco,

CEP 50630-970, CP 7462, Recife, PE, Brasil,

e-mails: Ihazin@ufpe.br; aalmeida@ufpe.br; carolmm@ufpe.br

Agradecimentos: O presente trabalho foi realizado com o apoio da CAPES - Coordenação de Aperfeiçoamento de Pessoal de Nível Superior e do CNPq - Conselho Nacional de Desenvolvimento Científico e Tecnológico.

Recebido em 03/4/2006

Aceito em 16/10/2007 
